\title{
Model-Based Pseudo-Quad-Pol Reconstruction from Compact Polarimetry and Its Application to Oil-Spill Observation
}

\author{
Junjun Yin, ${ }^{1,2}$ Wooil Moon, ${ }^{1}$ and Jian Yang ${ }^{2}$ \\ ${ }^{1}$ Geophysics, Faculty of Environment, Earth and Resources, University of Manitoba, Room 328, Wallace Building, Winnipeg, \\ $M B$, Canada R3T 2N2 \\ ${ }^{2}$ Department of Electronic Engineering, Tsinghua University, Room 8-302, Rohm Building, Beijing 100084, China
}

Correspondence should be addressed to Junjun Yin; yinjj07@gmail.com

Received 30 December 2014; Revised 16 March 2015; Accepted 15 April 2015

Academic Editor: Mike McShane

Copyright ( $(2015$ Junjun Yin et al. This is an open access article distributed under the Creative Commons Attribution License, which permits unrestricted use, distribution, and reproduction in any medium, provided the original work is properly cited.

\begin{abstract}
Compact polarimetry is an effective imaging mode for wide area observation, especially for the open ocean. In this study, we propose a new method for pseudo-quad-polarization reconstruction from compact polarimetry based on the three-component decomposition. By using the decomposed powers, the reconstruction model is established as a power-weighted model. Further, the phase of the copolarized correlation is taken into consideration. The phase of double-bounce scattering is closer to $\pi$ than to 0 , while the phase of surface scattering is closer to 0 than to $\pi$. By considering the negative (double-bounce reflection) and positive (surface reflection) copolarized correlation, the reconstruction model for full polarimetry has a good consistency with the real polarimetric SAR data. L-band ALOS/PALSAR-1 fully polarimetric data acquired on August 27, 2006, over an oil-spill area are used for demonstration. Reconstruction performance is evaluated with a set of typical polarimetric oil-spill indicators. Quantitative comparison is given. Results show that the proposed model-based method is of great potential for oil-spill observation.
\end{abstract}

\section{Introduction}

Crude oil and petroleum products pollution has severe impact on the marine environment. It results in large scale damage to local ecosystem, presenting potential enormous harm to deep ocean and coastal fisheries, wildlife, and regeneration. Rapid increase in oil-spill pollution is primarily due to the increased human ocean activities, which increase the risk of oil-spillage from both ship/oil-platform/pipeline accidents and routine ship operations like tank washing and engine effluent discharge. One of the mostly used instruments for ocean surveillance is synthetic aperture radar (SAR), which has the all-day and all-weather imaging capability and is sensitive to the ocean surface capillary-gravity waves [1-3]. Polarimetric SAR (Pol-SAR) offers multichannel polarimetric information, and the fully or quad polarimetric (quadpol) SAR system allows the complete backscattering characterization for scatterers. However, the fully polarimetric imaging mode suffers from system complexity, data volume, and the limited imaging coverage compared to SAR systems which use a single polarization for transmission [4]. In 2005, a polarimetric imaging concept was proposed and generally well known as compact polarimetry (CP) [5]. At present, both the Indian RISAT- 1 and JAXA ALOS/PALSAR- 2 can provide the $\mathrm{CP}$ mode. In the future, the $\mathrm{CP}$ mode will be prepared for launches of other Earth Observation (EO) satellites, for example, SAOCOM-1 and Radarsat Constellation Mission (RCM).

The compact SAR data can be processed in two manners: the first one is to reconstruct pseudo-quad-polarization data from compact polarimetry, and then many quad-pol methods can be applied to the reconstructed data [5-9] for various applications; the second one is to extract target scattering information directly from compact data $[4,10$, 11]. In this study, we focus on the reconstruction method. In the multipolarization reconstruction, two assumptions are very essential. One is the well-known reflection symmetry assumption, and the other is the polarization state extrapolation model, that is, the reconstruction model. There mainly exist five reconstruction methods in the literature. 
Studies in [5-8] are based on reflection symmetry to estimate the pseudocovariance matrix. The main difference between these methods is that they adopt different reconstruction model parameter $N . N$ is determined by either theoretical assumptions or empirical tests. In [9], we developed a method which can be applied to the nonreflection case based on the four-component decomposition. However, the fourcomponent decomposition is not applied well to describe the ocean surface where reflection symmetry always holds for most sea state conditions. Methods proposed in [7] by Collins et al. and in [8] by Li et al. are designed for ocean target detection. However, both methods are all empirical methods which need the prior fully polarimetric SAR data to fit the model parameter $N$. The nonlinear regression is usually used to determine the best curve fitting parameters. The difference between these two methods is that Li's model parameter $N$ needs updating when performing the iteration, while Collins's model parameter $N$ is a constant only relating to the incidence angle. Moreover, in [7], Collins et al. proposed to use a negative exponential curve to estimate $N$ for the C-band RADARSAT-2 data, while in [8], Collins's model parameter is fitted with a polynomial function for the $L$-band UAVSAR data. This implies that, for different SAR sensors, imaging geometry, and sea conditions, the fitting curves would probably like to vary greatly. This is not beneficial for practical applications. If the selected objective curve is not a best fit of the acquired data, then reconstruction performance can be expected to deteriorate.

In this paper, a model-based reconstruction method is proposed to extract the quad-pol information from compact polarimetry for oil-spill observation. By assuming a three-component decomposition for backscatter of the ocean surface, the model parameter $N$ is estimated based on the decomposed scattering powers. L-band ALOS/PALSAR-1 fully polarimetric data are used for demonstration. $L$-band polarimetric SAR data, especially the satellite data, are not widely investigated for oil-spill detection due to its long wavelength. Since $L$-band polarimetric SAR satellites are in operation (e.g., ALOS/PALSAR-2) and to be planned for future missions, it is necessary to explore the performance of $L$-band compact polarimetric data for oil-spill observation. Performance of the proposed method is evaluated in terms of a set of polarimetric indicators which are widely used for oil-spill observation in the literature [1]. The organization of the rest of this paper is as follows. In Section 2, we briefly introduce the $L$-band test data. In Section 3, the proposed reconstruction method is presented. Section 4 gives the experiments and comparison; and conclusions are given in Section 5.

\section{Test Data and Preprocessing}

L-band ALOS/PALSAR-1 fully polarimetric data acquired over the southern-eastern coast of Negros Island on August 27, 2006, are used for demonstration. These data are relevant to an oil-spill accident happened on August 11, 2006 [12]. A subset of the image is shown in Figure 1, from which we can see dark areas of oil-slicks. The fully polarimetric data are

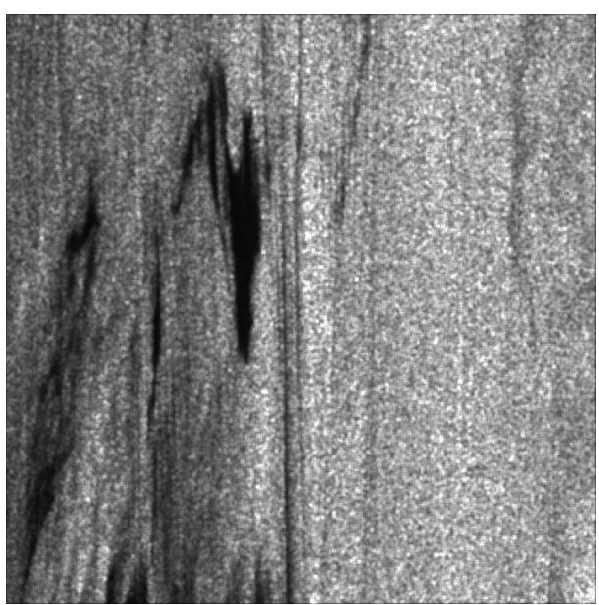

FIGURE 1: Span image of the test data, acquired on August 27, 2006. ALOS/PALSAR-1 site identification is ALPSRP031440190. The dark area is an oil-slick [12].

received in the single look complex (SLC) product format. This subset contains $1088 \times 1088$ pixels. Before performing analysis, a boxcar filter with a window size 7 has been applied to reduce the speckle. Linear compact polarimetric SAR data are synthesized from the fully polarimetric SAR data according to its specific scattering matrix, which will be introduced in Section 3.

\section{Pseudo-Quad-Polarization Reconstruction from Linear Compact Polarimetry}

3.1. Quad-Pol Data and Three-Component Decomposition. In the linear horizontal $(\mathrm{H})$ and vertical $(\mathrm{V})$ polarization base, the scattering vector is defined as shown in the following equation under the reciprocity theorem for the monostatic backscattering case:

$$
\vec{k}_{L}=\left[\begin{array}{lll}
S_{\mathrm{HH}} & \sqrt{2} S_{\mathrm{HV}} & S_{\mathrm{VV}}
\end{array}\right]^{T},
$$

where $S_{\mathrm{HV}}$ denotes $\mathrm{V}$ in transmission and $\mathrm{H}$ in reception. For the multilook case, the covariance matrix is shown in the following equation to represent the average scattering property:

$$
\begin{aligned}
\mathbf{C} & =\left\langle\vec{k}_{L} \vec{k}_{L}^{\mathrm{H}}\right\rangle \\
& =\left[\begin{array}{ccc}
\left\langle\left|S_{\mathrm{HH}}\right|^{2}\right\rangle & \sqrt{2}\left\langle S_{\mathrm{HH}} S_{\mathrm{HV}}^{*}\right\rangle & \left\langle S_{\mathrm{HH}} S_{\mathrm{VV}}^{*}\right\rangle \\
\sqrt{2}\left\langle S_{\mathrm{HV}} S_{\mathrm{HH}}^{*}\right\rangle & 2\left\langle\left|S_{\mathrm{HV}}\right|^{2}\right\rangle & \sqrt{2}\left\langle S_{\mathrm{HV}} S_{\mathrm{VV}}^{*}\right\rangle \\
\left\langle S_{\mathrm{VV}} S_{\mathrm{HH}}^{*}\right\rangle & \sqrt{2}\left\langle S_{\mathrm{VV}} S_{\mathrm{HV}}^{*}\right\rangle & \left\langle\left|S_{\mathrm{VV}}\right|^{2}\right\rangle
\end{array}\right],
\end{aligned}
$$

where $\langle\cdots\rangle$ and ${ }^{\mathrm{H}}$ denote spatial average and matrix transpose conjugate, respectively and ${ }^{*}$ denotes complex conjugation. Polarimetric target decomposition has been studied by many researchers [13-15]. Model-based decomposition aims to decompose the radar received backscattered energy into 
several components according to scattering models. Threecomponent decomposition [13] can be written as follows:

$$
\mathbf{C}=f_{s}\left[\mathbf{C}_{s}\right]+f_{d}\left[\mathbf{C}_{d}\right]+f_{v}\left[\mathbf{C}_{v}\right]
$$

where $f_{s}, f_{d}$, and $f_{v}$ are decomposed parameters to be determined corresponding to the surface, double-bounce, and volume scattering models, respectively. These three scattering models are given by

$$
\begin{aligned}
& {\left[\mathbf{C}_{s}\right]=\left[\begin{array}{ccc}
|\beta|^{2} & 0 & \beta \\
0 & 0 & 0 \\
\beta^{*} & 0 & 1
\end{array}\right],} \\
& {\left[\mathbf{C}_{d}\right]=\left[\begin{array}{ccc}
|\alpha|^{2} & 0 & \alpha \\
0 & 0 & 0 \\
\alpha^{*} & 0 & 1
\end{array}\right],} \\
& {\left[\mathbf{C}_{v}\right]=\left[\begin{array}{ccc}
1 & 0 & b \\
0 & 1-b & 0 \\
b & 0 & 1
\end{array}\right], \quad b \in\left[\begin{array}{ll}
0 & 1)
\end{array}\right.}
\end{aligned}
$$

where $\beta$ and $\alpha$ are model parameters with $\arg (\beta) \approx 0$ and $\arg (\alpha) \approx \pm \pi$. Different three-component decomposition methods are generally developed by modifying the volume scattering model $\left[\mathbf{C}_{v}\right] .\left[\mathbf{C}_{v}\right]$ presented in (4) is a general volume scattering model exhibiting scattering azimuthal symmetry. In Freeman and Durden's 3-component decomposition $[13,15], b$ is set to $1 / 3$. Effects of the parameter $b$ on scattering powers and on the compact reconstruction model will be discussed in the next section. If we let $b=0$, by relating (3) and (4) to (2), the backscattered energy span can be expanded into scattering powers as shown in the following:

$$
\begin{aligned}
P_{s} & =f_{s}\left(1+|\beta|^{2}\right), \\
P_{d} & =f_{d}\left(1+|\alpha|^{2}\right), \\
P_{v} & =3 f_{v}=6\left\langle\left|S_{\mathrm{HV}}\right|^{2}\right\rangle, \\
\operatorname{span} & =\left\langle\left|S_{\mathrm{HH}}\right|^{2}\right\rangle+2\left\langle\left|S_{\mathrm{HV}}\right|^{2}\right\rangle+\left\langle\left|S_{\mathrm{VV}}\right|^{2}\right\rangle \\
& =P_{s}+P_{d}+P_{v},
\end{aligned}
$$

where $P_{s}, P_{d}$, and $P_{v}$ are the decomposed powers. Since this decomposition has 5 unknowns with 4 equations, the sign of $\operatorname{Re}\left(\left\langle S_{\mathrm{HH}} S_{\mathrm{VV}}^{*}\right\rangle\right)$ is usually used to determine whether surface reflection or double-bounce reflection is dominant [13]. Specifically, if $\operatorname{Re}\left(\left\langle S_{\mathrm{HH}} S_{\mathrm{VV}}^{*}\right\rangle\right)>0$, then let $\alpha=-1$ (indicating a dominant surface reflection); if $\operatorname{Re}\left(\left\langle S_{\mathrm{HH}} S_{\mathrm{VV}}^{*}\right\rangle\right)<$ 0 , then let $\beta=1$ (indicating a dominant double-bounce reflection), where $\operatorname{Re}(\cdots)$ indicates the real part of a complex number.

\subsection{Compact Polarimetry and the Proposed Model-Based Reconstruction Model}

3.2.1. Linear $\pi / 4$ Compact Data. The compact system measures a projection of the complex scattering matrix $\mathbf{S}$ onto a transmitted electromagnetic field $\vec{E}_{i}$. When transmitting a slant-linear polarization oriented at $45^{\circ}$, the received electromagnetic wave is given by

$$
\begin{aligned}
\vec{E}_{r} & =\overrightarrow{\mathbf{S E}}_{i}=\frac{1}{\sqrt{2}}\left[\begin{array}{ll}
S_{\mathrm{HH}} & S_{\mathrm{HV}} \\
S_{\mathrm{HV}} & S_{\mathrm{VV}}
\end{array}\right]\left[\begin{array}{l}
1 \\
1
\end{array}\right] \\
& =\frac{1}{\sqrt{2}}\left[\begin{array}{l}
S_{\mathrm{HH}}+S_{\mathrm{HV}} \\
S_{\mathrm{VV}}+S_{\mathrm{HV}}
\end{array}\right] .
\end{aligned}
$$

$\vec{E}_{r}$ is called the Jones vector of the scattered wave. Equation (6) gives the scattering vector for the $\pi / 4$ compact mode. We usually use the wave covariance matrix, defined in (7), to analyze the target average scattering property. From (7), it is seen that for the $\pi / 4$ mode, the covariance matrix $\mathbf{C}_{\pi / 4}$ can be written as a sum of three parts, that is, a copolarized information part, a cross-polarization part, and the residue part:

$$
\begin{aligned}
\mathbf{C}_{\pi / 4} & =\left\langle\vec{E}_{r} \vec{E}_{r}^{\mathrm{H}}\right\rangle=\left[\begin{array}{ll}
C_{11} & C_{12} \\
C_{21} & C_{22}
\end{array}\right] \\
& =\frac{1}{2}\left\{\left[\begin{array}{cc}
\left\langle\left|S_{\mathrm{HH}}\right|^{2}\right\rangle & \left\langle S_{\mathrm{HH}} S_{\mathrm{VV}}^{*}\right\rangle \\
\left\langle S_{\mathrm{VV}} S_{\mathrm{HH}}^{*}\right\rangle & \left\langle\left|S_{\mathrm{VV}}\right|^{2}\right\rangle
\end{array}\right]+\left\langle\left|S_{\mathrm{HV}}\right|^{2}\right\rangle\left[\begin{array}{ll}
1 & 1 \\
1 & 1
\end{array}\right]\right. \\
& \left.+\left[\begin{array}{cc}
2 \operatorname{Re}\left(\left\langle S_{\mathrm{HH}} S_{\mathrm{HV}}^{*}\right\rangle\right) & \left\langle S_{\mathrm{HH}} S_{\mathrm{HV}}^{*}\right\rangle+\left\langle S_{\mathrm{HV}} S_{\mathrm{VV}}^{*}\right\rangle \\
\left\langle S_{\mathrm{HV}} S_{\mathrm{HH}}^{*}\right\rangle+\left\langle S_{\mathrm{VV}} S_{\mathrm{HV}}^{*}\right\rangle & 2 \operatorname{Re}\left(\left\langle S_{\mathrm{VV}} S_{\mathrm{HV}}^{*}\right\rangle\right)
\end{array}\right]\right\} .
\end{aligned}
$$

By assuming reflection symmetry, the last submatrix in (7) is usually omitted; that is, the terms involving products of copolarized and cross-polarized terms are ignored. For most terrain types, the omitted part is much smaller than the others. Reflection symmetry is also valid for the ocean surface. By this means, there are only 4 parameters left in $\mathbf{C}_{\pi / 4}$, that is, $\left\langle\left|S_{\mathrm{HH}}\right|^{2}\right\rangle,\left\langle\left|S_{\mathrm{VV}}\right|^{2}\right\rangle,\left\langle S_{\mathrm{HH}} S_{\mathrm{VV}}^{*}\right\rangle$, and $\left\langle\left|S_{\mathrm{HV}}\right|^{2}\right\rangle$. In the reconstruction of pseudo-quad-pol information, these parameters need to be estimated. There are 4 unknowns, but only 3 equations can be obtained from (7), so an extra reconstruction model is needed to relate these parameters. In the literature, there exist four main reconstruction modes [58] which have the following general form:

$$
\frac{\left\langle\left|S_{\mathrm{HV}}\right|^{2}\right\rangle}{\left\langle\left|S_{\mathrm{HH}}\right|^{2}\right\rangle+\left\langle\left|S_{\mathrm{VV}}\right|^{2}\right\rangle}=\frac{1-|\rho|}{N},
$$

where $\rho$ is the copolarization correlation coefficient, defined by $\rho=\left\langle S_{\mathrm{HH}} S_{\mathrm{VV}}^{*}\right\rangle / \sqrt{\left\langle\left|S_{\mathrm{HH}}\right|^{2}\right\rangle\left\langle\left|S_{\mathrm{VV}}\right|^{2}\right\rangle}$ and $N$ is the model parameter which is different for different reconstruction algorithms. Souyris et al. first proposed the reconstruction model using $N=4$. We calculate both $\left\langle\left|S_{\mathrm{HV}}\right|^{2}\right\rangle /\left(\left\langle\left|S_{\mathrm{HH}}\right|^{2}\right\rangle+\right.$ $\left.\left\langle\left|S_{\mathrm{VV}}\right|^{2}\right\rangle\right)$ and $\rho$ for the scattering models in (4), as shown 
in (9). We could observe that Souyris's model satisfies all these scattering behaviours when the scattering models are considered separately:

$$
\begin{gathered}
\mathrm{C}_{s}: \frac{\left\langle\left|S_{\mathrm{HV}}\right|^{2}\right\rangle}{\left(\left\langle\left|S_{\mathrm{HH}}\right|^{2}\right\rangle+\left\langle\left|S_{\mathrm{VV}}\right|^{2}\right\rangle\right)}=0, \quad \rho=1, \\
\mathrm{C}_{d}: \frac{\left\langle\left|S_{\mathrm{HV}}\right|^{2}\right\rangle}{\left(\left\langle\left|S_{\mathrm{HH}}\right|^{2}\right\rangle+\left\langle\left|S_{\mathrm{VV}}\right|^{2}\right\rangle\right)}=0, \quad \rho=-1, \\
\mathrm{C}_{v}: \frac{\left\langle\left|S_{\mathrm{HV}}\right|^{2}\right\rangle}{\left(\left\langle\left|S_{\mathrm{HH}}\right|^{2}\right\rangle+\left\langle\left|S_{\mathrm{VV}}\right|^{2}\right\rangle\right)}=\frac{1-b}{4}, \quad \rho=b .
\end{gathered}
$$

We review Souyris's model from a new perspective. A scattering process is actually a mixture of scattering processes of elemental scatterers in a resolution cell. Backscatter is affected by all the elemental scatterers. If we assume a threecomponent decomposition and that each component contributes to the backscattered features in the scale of its power proportion, then Souyris's model can be taken as a powerweighted model as long as the phase of $\rho$ is not considered; that is, $|\rho|$ is used in the reconstruction model. Souyris's model does not distinguish the difference of the copolarized correlation coefficients of the surface and double-bounce reflections. The typical single-bounce reflection has a value of $\rho$ equal to 1 , while the typical double-bounce reflection has a value of $\rho$ equal to -1 . By taking into consideration that the copolarized correlation coefficient $\rho$ can be either positive or negative, we propose a new reconstruction model based on three-component decomposition, given in the next section.

3.2.2. The Proposed Reconstruction Model. From (9), we can establish a model-based power-weighted reconstruction model. By assuming that the backscattered energy is a sum of the surface scattering power $P_{s}$, double-bounce scattering power $P_{d}$, and volume scattering power $P_{v}$, the following relationship can be obtained:

$$
\begin{aligned}
\mathbf{C}_{s}+\mathbf{C}_{d}+\mathbf{C}_{v} \\
\Longrightarrow\left\{\begin{array}{c}
\frac{\left\langle\left|S_{\mathrm{HV}}\right|^{2}\right\rangle}{\left\langle\left|S_{\mathrm{HH}}\right|^{2}\right\rangle+\left\langle\left|S_{\mathrm{VV}}\right|^{2}\right\rangle}=\frac{1-b}{4} \frac{P_{v}}{\operatorname{span}} \\
\frac{1-\rho}{4}=\frac{2 P_{d}+(1-b) P_{v}}{4 \operatorname{span}} .
\end{array}\right.
\end{aligned}
$$

For natural distributed targets, $\rho$ is a complex number, so $|\rho|$ is still used in the reconstruction [5]. However, in order to preserve the positive or negative correlation property (i.e., the copolarized phase difference is either closer to 0 or closer to $\pi)$ of the surface or double-bounce scattering, $\operatorname{Re}\left(\left\langle S_{\mathrm{HH}} S_{\mathrm{VV}}^{*}\right\rangle\right)$ is incorporated in the modified model as follows:

$$
\begin{aligned}
& \frac{\left\langle\left|S_{\mathrm{HV}}\right|^{2}\right\rangle}{\left\langle\left|S_{\mathrm{HH}}\right|^{2}\right\rangle+\left\langle\left|S_{\mathrm{VV}}\right|^{2}\right\rangle} \\
& \quad=\frac{1-\operatorname{sgn}\left(\operatorname{Re}\left(\left\langle S_{\mathrm{HH}} S_{\mathrm{VV}}^{*}\right\rangle\right)\right)|\rho|}{4} \frac{(1-b) P_{v}}{2 P_{d}+(1-b) P_{v}},
\end{aligned}
$$

where $\operatorname{sgn}(\cdots)$ is a signum function. In order to find a suitable parameter $b$ for the reconstruction, we test the equality of the two sides of (11). When $b$ varies in the interval [ $\left[\begin{array}{ll}0 & 1\end{array}\right)$, the proportion of scattering powers decomposed from (3) varies accordingly. Using the test data for illustration, the polynomial curves fitting to the two sides of (11) is shown in Figure 2(a), from which it is observed that the fitted curve with $b=0$ is much closer to the diagonal line than the other fitted lines. It should be noted that $b$ should not be larger than $\left(\operatorname{span}-6\left\langle\left|S_{\mathrm{HV}}\right|^{2}\right\rangle\right) /\left(\operatorname{span}-2\left\langle\left|S_{\mathrm{HV}}\right|^{2}\right\rangle\right)$; otherwise there will occur with negative decomposed powers. Further, when $b$ increases from 0 , the number of the pixels falling in the plane of Figure 2(a) decreases. This variation is shown in Figure 2(b), which means that the number of effective pixels decreases with $b$ increasing. Thus in order to make sure that (11) is valid and effective for most pixels and ensures the equality, $b$ is set to 0 .

Then the proposed reconstruction model is then obtained as

$$
\frac{\left\langle\left|S_{\mathrm{HV}}\right|^{2}\right\rangle}{\left\langle\left|S_{\mathrm{HH}}\right|^{2}\right\rangle+\left\langle\left|S_{\mathrm{VV}}\right|^{2}\right\rangle}=\frac{1-\mathrm{sg} \cdot|\rho|}{4} \frac{P_{v}}{2 P_{d}+P_{v}}
$$

where sg $=\operatorname{sgn}\left(\operatorname{Re}\left(\left\langle S_{\mathrm{HH}} S_{\mathrm{VV}}^{*}\right\rangle\right)\right)$. Using the test data, Figure 3 compares the present reconstruction model with Souyris's model. It is observed that the developed model is more consistent with the real polarimetric SAR data; that is, the points are scattered much closer to the diagonal line with the proposed model.

Next we need to consider how to approximate $\operatorname{sgn}\left(\operatorname{Re}\left(\left\langle S_{\mathrm{HH}} S_{\mathrm{VV}}^{*}\right\rangle\right)\right), P_{d}$, and $P_{v}$ in the linear $\pi / 4$ compact polarimetric mode. From the coherency matrix shown in (7), we notice that when assuming reflection symmetry, the sign of $\operatorname{Re}\left(\left\langle S_{\mathrm{HH}} S_{\mathrm{VV}}^{*}\right\rangle\right)$ is only affected by $\left\langle\left|S_{\mathrm{HV}}\right|^{2}\right\rangle$. For the ocean surface, the high copolarized correlation is always expected to make the copolarized terms larger than the cross-polarized term [12]. Hence, we use $\operatorname{Re}\left(C_{12}\right)$ to determine whether the backscatter process is dominated by surface or by double-bounce scattering. We use the whole data of ALPSRP031440190, which contains $18432 \times 1088$ pixels, to test the consistency between areas determined by $\operatorname{Re}\left(C_{12}\right)>0$ and $\operatorname{Re}\left(\left\langle S_{\mathrm{HH}} S_{\mathrm{VV}}^{*}\right\rangle\right)>0$. The overall agreement is $96.7 \%$. Thus, this principle is valid and reasonable to determine which scattering mechanism dominates the backscatter of the CP mode, at least for the $L$-band PALSAR data.

From the covariance scattering models in (4), the corresponding compact scattering models can then be synthesized as shown in (13), where the parameter $b$ in $\mathbf{C}_{v}$ is set to 0 :

$$
\begin{aligned}
& {\left[\mathbf{C}_{s}\right]_{\mathrm{CP}}=\left[\begin{array}{cc}
|\beta|^{2} & \beta \\
\beta^{*} & 1
\end{array}\right] ;} \\
& {\left[\mathbf{C}_{d}\right]_{\mathrm{CP}}=\left[\begin{array}{cc}
|\alpha|^{2} & \alpha \\
\alpha^{*} & 1
\end{array}\right] ;} \\
& {\left[\mathbf{C}_{v}\right]_{\mathrm{CP}}=\left[\begin{array}{ll}
1.5 & 0.5 \\
0.5 & 1.5
\end{array}\right] .}
\end{aligned}
$$




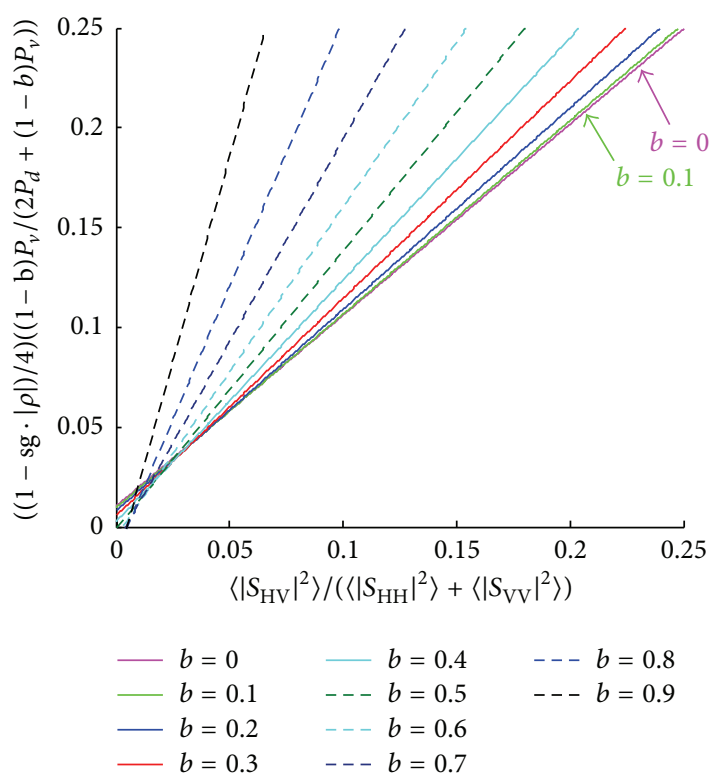

(a)

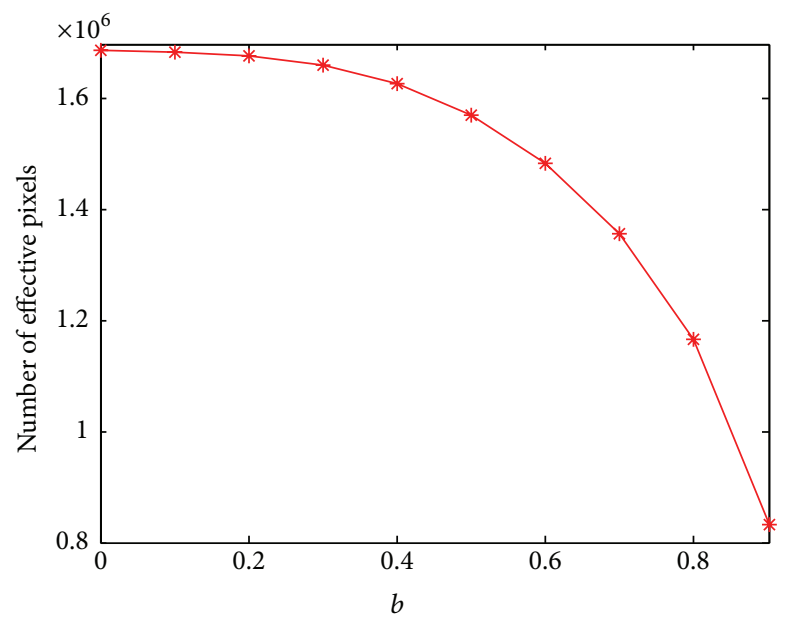

(b)

FIGURE 2: (a) First order polynomial curve fittings of the left side (shown on the abscissa) and the right side (shown on the ordinate) of (11) by varying the parameter $b$ by using the test data. (b) Variation of $b$ versus the number of the pixels falling in the plane in (a).

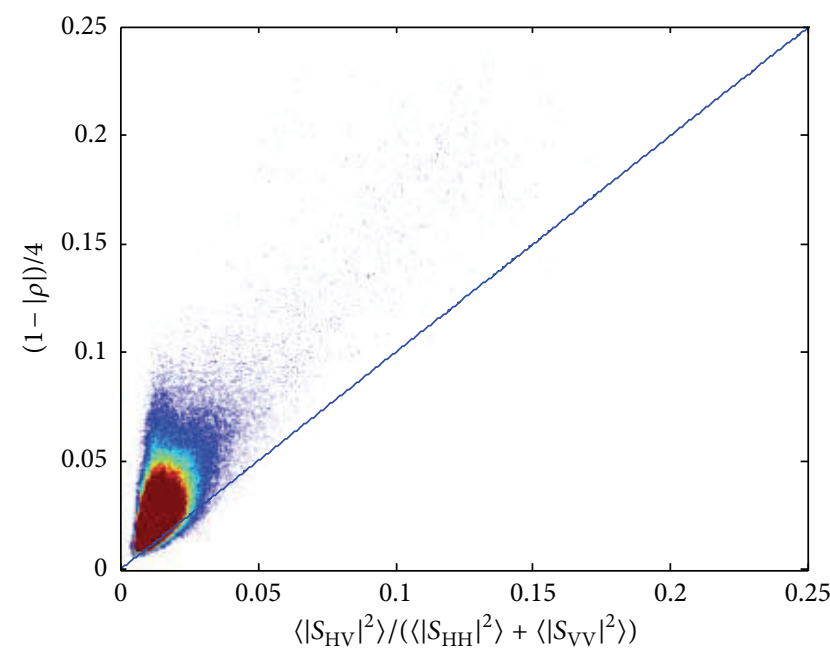

(a)

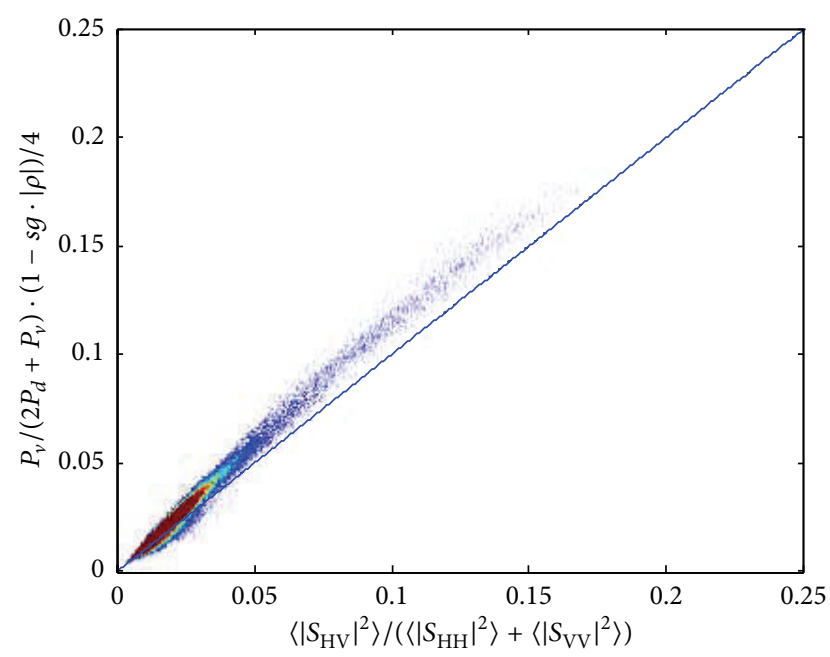

(b)

FIGURE 3: Comparison of reconstruction models. (a) Souyris's model. (b) The proposed model.

$\left[\mathbf{C}_{s}\right]_{\mathrm{CP}},\left[\mathbf{C}_{d}\right]_{\mathrm{CP}}$, and $\left[\mathbf{C}_{v}\right]_{\mathrm{CP}}$ are the compact covariance matrices of surface, double-bounce, and volume scattering, respectively. Then the compact covariance matrix can be expanded as

$$
\mathbf{C}_{\pi / 4}=f_{s c}\left[\mathbf{C}_{s}\right]_{\mathrm{CP}}+f_{d c}\left[\mathbf{C}_{d}\right]_{\mathrm{CP}}+f_{v c}\left[\mathbf{C}_{v}\right]_{\mathrm{CP}}
$$

where $f_{s c}, f_{d c}$, and $f_{v c}$ are the expansion coefficients. From (14), we have 3 equations with 5 unknowns. It is noted from (5) that, for the fully polarimetric case, we have $f_{v}=$
$2\left\langle\left|S_{\mathrm{HV}}\right|^{2}\right\rangle$. The backscattered energy in full polarimetry is double that of compact polarimetry. Hence, the decomposed coefficient $f_{v c}$ is expected to be proportionally reduced. Let $f_{v c}=\left\langle\left|S_{\mathrm{HV}}\right|^{2}\right\rangle \cdot\left\langle\left|S_{\mathrm{HV}}\right|^{2}\right\rangle$ is a parameter needed to be estimated in the reconstruction. If $\left\langle\left|S_{\mathrm{HV}}\right|^{2}\right\rangle$ is retained to be determined later, then (14) can be solved in a similar way as that of three-component decomposition. When $\operatorname{Re}\left(C_{12}\right)$ is positive, we decide that surface scattering is dominant and let $\alpha=$ -1 . When $\operatorname{Re}\left(C_{12}\right)$ is negative, we decide that double-bounce 
scattering is dominant and let $\beta=1$. Finally, the surface scattering power $P_{s c}$ and the double-bounce scattering power $P_{d c}$ can be obtained as follows:

$$
\begin{gathered}
X=C_{11}-1.5 f_{v c} ; \\
Y=C_{22}-1.5 f_{v c} ; \\
Z=C_{12}-0.5 f_{v c} ; \\
P_{d c}=2 \frac{X Y-|Z|^{2}}{X+Y+2 \operatorname{Re}(Z)}, \quad \text { if } \operatorname{Re}\left(C_{12}\right)>0, \alpha=-1 \\
\text { or } P_{s c}=2 \frac{X Y-|Z|^{2}}{X+Y-2 \operatorname{Re}(Z)}, \quad \text { if } \operatorname{Re}\left(C_{12}\right)<0, \beta=1 .
\end{gathered}
$$

After $P_{d c}$ or $P_{s c}$ is obtained, for the above two cases, $P_{s c}$ in the case $\operatorname{Re}\left(C_{12}\right)>0$ and $P_{d c}$ in the case $\operatorname{Re}\left(C_{12}\right)<0$ can be directly obtained from $P_{s c}=\operatorname{span}_{c}-P_{d c}-P_{v c}$ and $P_{d c}=\operatorname{span}_{c}-P_{s c}-P_{v c}$, respectively, where $\operatorname{span}_{c}=C_{11}+$ $C_{22}$ is the total backscattered power. The volume scattering power $P_{v c}=3 f_{v c}=3\left\langle\left|S_{\mathrm{HV}}\right|^{2}\right\rangle$ will be estimated during the iteration introduced in the next section. Note that this three-component decomposition only serves for the purpose of pseudo-quad-pol data reconstruction. It is quite different from other compact target decompositions $[4,11]$.

3.3. Reconstruction Algorithm. The iteration approach is employed to solve the nonlinear system [5-9]. The iteration procedure is detailed as follows.

Step 0. Initialization. Consider

$$
\begin{aligned}
N_{(0)} & =4 \\
\rho_{(0)} & =\frac{C_{12}}{\sqrt{C_{11} C_{22}}}, \\
f_{v c(0)} & =\left(C_{11}+C_{22}\right) \frac{1-\mathrm{sg} \cdot\left|\rho_{(0)}\right|}{N_{(0)} / 2+1-\mathrm{sg} \cdot\left|\rho_{(0)}\right|} .
\end{aligned}
$$

Step 1. Iteration. Consider

$$
\begin{gathered}
X=C_{11}-1.5 f_{v c(i)} ; \\
Y=C_{22}-1.5 f_{v c(i)} ; \\
Z=C_{12}-0.5 f_{v c(i)} ; \\
P_{d c(i)}=2 \frac{X Y-|Z|^{2}}{X+Y+2 \operatorname{Re}(Z)} \\
N_{(i+1)}=4 \frac{2 P_{d c(i)}+3 f_{v c(i)}}{3 f_{v c(i)}}
\end{gathered}
$$

$$
\text { if } \operatorname{Re}\left(C_{12}\right)>0
$$

$$
\begin{gathered}
\text { or } P_{s c(i)}=2 \frac{X Y-|Z|^{2}}{X+Y-2 \operatorname{Re}(Z)} \\
N_{(i+1)}=4 \frac{2 \operatorname{span}_{c}-2 P_{s c(i)}-3 f_{v c(i)}}{3 f_{v c(i)}} \\
\rho_{(i+1)}=\frac{C_{12}-f_{v c(i)} / 2}{\sqrt{\left(C_{11}-f_{v c(i)} / 2\right)\left(C_{22}-f_{v c(i)} / 2\right)}} \\
f_{v c(i+1)}=\left(C_{11}+C_{22}\right) \frac{1-\operatorname{sg} \cdot\left|\rho_{(i+1)}\right|}{N_{(i+1)} / 2+1-\operatorname{sg} \cdot\left|\rho_{(i+1)}\right|},
\end{gathered}
$$

where sg $=\operatorname{sgn}\left(\operatorname{Re}\left(C_{12}\right)\right)$ and $i=0,1,2, \ldots$ is the iterations number. Given a converged value for $f_{v c}$, where $\left\langle\left|S_{\mathrm{HV}}\right|^{2}\right\rangle=$ $f_{v c}$, the pseudo-quad-pol covariance matrix is estimated as

$$
\begin{aligned}
& \mathbf{C}_{\text {pseudo-quad }} \\
& =\left[\begin{array}{ccc}
2 C_{11}-\left\langle\left|S_{\mathrm{HV}}\right|^{2}\right\rangle & 0 & 2 C_{12}-\left\langle\left|S_{\mathrm{HV}}\right|^{2}\right\rangle \\
0 & 2\left\langle\left|S_{\mathrm{HV}}\right|^{2}\right\rangle & 0 \\
2 C_{12}^{*}-\left\langle\left|S_{\mathrm{HV}}\right|^{2}\right\rangle & 0 & 2 C_{22}-\left\langle\left|S_{\mathrm{HV}}\right|^{2}\right\rangle
\end{array}\right] .
\end{aligned}
$$

\section{Experiments}

In experiments, by comparing with the method proposed by Souyris et al., we validate the proposed model-based method in the following aspects: firstly, the reconstructed covariance matrix is evaluated in terms of the co- and crosspolarized elements; and secondly, several typical oil-slick indicators derived in full polarimetry are used to assess the performance of the proposed method. Souyris's method is selected for comparison mainly due to the reason that Souyris's method can also be seen as a power-weighted pseudo-quad-pol information reconstruction method. The only difference with the proposed method is that the negative or positive copolarized correlation property is considered in this study to discriminate between the surface and doublebounce scattering. Thus, the comparison results would clearly display the significance of the phase information in the improvement of reconstruction accuracy. The included fully polarimetric oil-spill indicators [1] are summarized in Table 1. Reconstruction results are shown in Table 2. From Table 2, it can be observed that the proposed method improves the reconstruction stably. In addition, this reconstruction method does not need prior fully polarimetric data to train the model parameter $N$.

\section{Conclusion}

In this study, we proposed a model-based reconstruction method for the linear $\pi / 4$ compact polarimetry. By taking the negative and positive copolarized correlation properties (negative for the double-bounce dominated case and positive for the surface dominated case) into consideration, 
TABLE 1: Oil-spill indicators of fully polarimetric SAR images.

Copolarized correlation coefficient $|\rho|=\left|\frac{\left\langle S_{\mathrm{HH}} S_{\mathrm{VV}}^{*}\right\rangle}{\sqrt{\left\langle\left|S_{\mathrm{HH}}\right|^{2}\right\rangle\left\langle\left|S_{\mathrm{VV}}\right|^{2}\right\rangle}}\right|$

Copolarized phase difference

$$
\mathrm{CPD}=\operatorname{angle}\left(\left\langle S_{\mathrm{HH}} S_{\mathrm{VV}}^{*}\right\rangle\right)
$$

Conformity coefficient

$$
u=\frac{2\left(\operatorname{Re}\left(\left\langle S_{\mathrm{HH}} S_{\mathrm{VV}}^{*}\right\rangle\right)-\left\langle\left|S_{\mathrm{HV}}\right|^{2}\right\rangle\right)}{\operatorname{span}}
$$

Circular polarization correlation

$$
\mathrm{CPC}=\left|\frac{\left\langle S_{\mathrm{RR}} S_{\mathrm{LL}}^{*}\right\rangle}{\sqrt{\left\langle\left|S_{\mathrm{RR}}\right|^{2}\right\rangle\left\langle\left|S_{\mathrm{LL}}\right|^{2}\right\rangle}}\right| \text {, where }\left\{\begin{array}{l}
S_{\mathrm{RR}}=\frac{\left(S_{\mathrm{HH}}-S_{\mathrm{VV}}+2 j S_{\mathrm{HV}}\right)}{2} \\
S_{\mathrm{LL}}=\frac{\left(S_{\mathrm{HH}}-S_{\mathrm{VV}}-2 j S_{\mathrm{HV}}\right)}{2}
\end{array}\right.
$$

$H$, alpha, $A$

Cloude-Pottier's decomposition

\begin{tabular}{|c|c|c|c|c|c|c|c|c|c|c|}
\hline & \multicolumn{2}{|c|}{$\left\langle\left|S_{\mathrm{HH}}\right|^{2}\right\rangle$} & \multicolumn{2}{|c|}{$\left\langle\left|S_{\mathrm{VV}}\right|^{2}\right\rangle$} & \multicolumn{2}{|c|}{$\left\langle\left|S_{\mathrm{HV}}\right|^{2}\right\rangle$} & \multicolumn{2}{|c|}{$|\rho|$} & \multicolumn{2}{|c|}{$\mathrm{CPD}\left({ }^{\circ}\right)$} \\
\hline & m. & Std. & m. & Std. & m. & Std. & $\mathrm{m}$. & Std. & $\mathrm{m}$. & Std. \\
\hline$N=4$ & 0.023 & 0.018 & 0.027 & 0.021 & 0.029 & 0.019 & 0.011 & 0.0175 & 0.861 & 6.544 \\
\hline \multirow[t]{3}{*}{ Proposed model } & 0.022 & 0.017 & 0.026 & 0.020 & 0.027 & 0.017 & 0.010 & 0.016 & 0.760 & 5.693 \\
\hline & \multicolumn{2}{|c|}{$u$} & \multicolumn{2}{|c|}{$\mathrm{CPC}$} & \multicolumn{2}{|c|}{$H$} & \multicolumn{2}{|c|}{ Alpha $\left(^{\circ}\right)$} & \multicolumn{2}{|c|}{$A$} \\
\hline & $\mathrm{m}$. & Std. & $\mathrm{m}$. & Std. & $\mathrm{m}$. & Std. & $\mathrm{m}$. & Std. & $\mathrm{m}$. & Std. \\
\hline$N=4$ & 0.040 & 0.033 & 0.316 & 0.128 & 0.070 & 0.040 & 0.303 & 1.034 & 0.324 & 0.124 \\
\hline Proposed model & 0.360 & 0.028 & 0.290 & 0.122 & 0.065 & 0.036 & 1.190 & 0.857 & 0.330 & 0.124 \\
\hline
\end{tabular}

TABLE 2: Mean (m.) and standard deviation (Std.) of the pseudo-quad-pol reconstruction errors for the covariance elements and the polarimetric parameters listed in Table $1 . N=4$ indicates the results of Souyris's model.

the proposed model is generally consistent with the real polarimetric SAR data. By employing a model-based compact target decomposition scheme, the reconstruction can be implemented via an iteration approach. In experiments, reconstruction performance is evaluated in terms of both coand cross-polarized elements and several typical polarimetric oil-spill indicators. Results showed that, by comparison with the Souyris method, the proposed method has a stable superior performance. In addition, this study investigated the capability of $L$-band satellite polarimetric SAR data for oilspill observation in compact mode, which has rarely appeared in the literature. In the future, detailed works will be carried out and the extension of this method to the circular compact mode will also be fully evaluated.

\section{Conflict of Interests}

The authors declare that there is no conflict of interests regarding the publication of this paper.

\section{Acknowledgments}

This work is partly supported by NSFC under Grant no. 41171317 and by the Research Foundation of Tsinghua University under Grant no. 20111080968. The first author, Junjun Yin, is supported while carrying on her research at the University of Manitoba by the NSERC Discovery Grant no. 7400 awarded to Wooil Moon.

\section{References}

[1] A. H. S. Solberg, "Remote sensing of ocean oil-spill pollution," IEEE Proceedings, vol. 100, no. 10, pp. 2931-2945, 2012.

[2] D.-J. Kim, W. M. Moon, and Y.-S. Kim, "Application of TerraSAR-X data for emergent oil-spill monitoring," IEEE Transactions on Geoscience and Remote Sensing, vol. 48, no. 2, pp. 852-863, 2010.

[3] D. L. Schuler and J.-S. Lee, "Mapping ocean surface features using biogenic slick-fields and SAR polarimetric decomposition techniques," IEE Proceedings: Radar, Sonar and Navigation, vol. 153, no. 3, pp. 260-270, 2006.

[4] F. J. Charbonneau, B. Brisco, R. K. Raney et al., "Compact polarimetry overview and applications assessment," Canadian Journal of Remote Sensing, vol. 36, no. 2, pp. S298-S315, 2010.

[5] J.-C. Souyris, P. Imbo, R. Fjørtoft, S. Mingot, and J.-S. Lee, "Compact polarimetry based on symmetry properties of geophysical media: the $\pi / 4$ mode," IEEE Transactions on Geoscience and Remote Sensing, vol. 43, no. 3, pp. 634-645, 2005.

[6] M. E. Nord, T. L. Ainsworth, J.-S. Lee, and N. J. S. Stacy, "Comparison of compact polarimetric synthetic aperture radar modes," IEEE Transactions on Geoscience and Remote Sensing, vol. 47, no. 1, pp. 174-188, 2009.

[7] M. J. Collins, M. Denbina, and G. Atteia, "On the reconstruction of quad-pol sar data from compact polarimetry data for ocean target detection," IEEE Transactions on Geoscience and Remote Sensing, vol. 51, no. 1, pp. 591-600, 2013.

[8] Y. Li, Y. Zhang, J. Chen, and H. Zhang, "Improved compact polarimetric SAR Quad-pol reconstruction algorithm for oil 
spill detection," IEEE Geoscience and Remote Sensing Letters, vol. 11, no. 6, pp. 1139-1142, 2014.

[9] J. Yin and J. Yang, "Multi-polarization reconstruction from compact polarimetry based on modified four-component scattering decomposition," Journal of Systems Engineering and Electronics, vol. 25, no. 3, Article ID 6850218, pp. 399-410, 2014.

[10] J. Yin, J. Yang, Z.-S. Zhou, and J. Song, “The extended bragg scattering model-based method for ship and oil-spill observation using compact polarimetric SAR," IEEE Journal of Selected Topics in Applied Earth Observations and Remote Sensing, 2014.

[11] S. R. Cloude, D. G. Goodenough, and H. Chen, "Compact decomposition theory," IEEE Geoscience and Remote Sensing Letters, vol. 9, no. 1, pp. 28-32, 2012.

[12] M. Migliaccio, A. Gambardella, F. Nunziata, M. Shimada, and O. Isoguchi, "The PALSAR polarimetric mode for sea oil slick observation," IEEE Transactions on Geoscience and Remote Sensing, vol. 47, no. 12, pp. 4032-4041, 2009.

[13] J. S. Lee and E. Pottier, Polarimetric Radar Imaging: From Basics to Applications, vol. 143 of Optical Science and Engineering Series, chapter 6, CRS Press, 2008.

[14] E. Pottier and S. R. Cloude, "Application of the H/A/ $\alpha$ polarimetric decomposition theorem for land classification," in Wideband Interferometric Sensing and Imaging Polarimetry, vol. 3120 of Proceedings of SPIE, pp. 132-143, San Diego, Calif, USA, July 1997.

[15] A. Freeman, "Fitting a two-component scattering model to polarimetric SAR data from forests," IEEE Transactions on Geoscience and Remote Sensing, vol. 45, no. 8, pp. 2583-2592, 2007. 

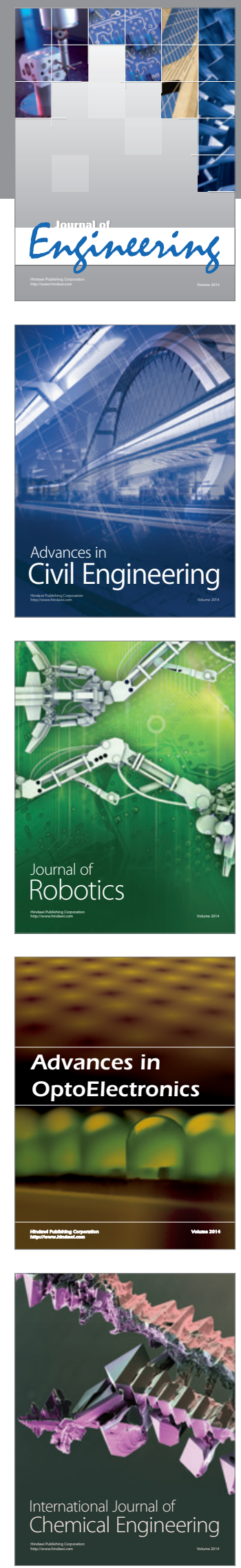

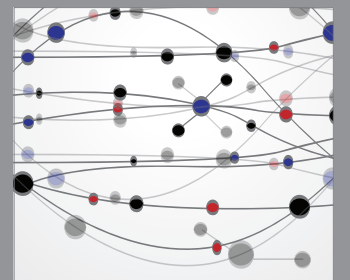

The Scientific World Journal
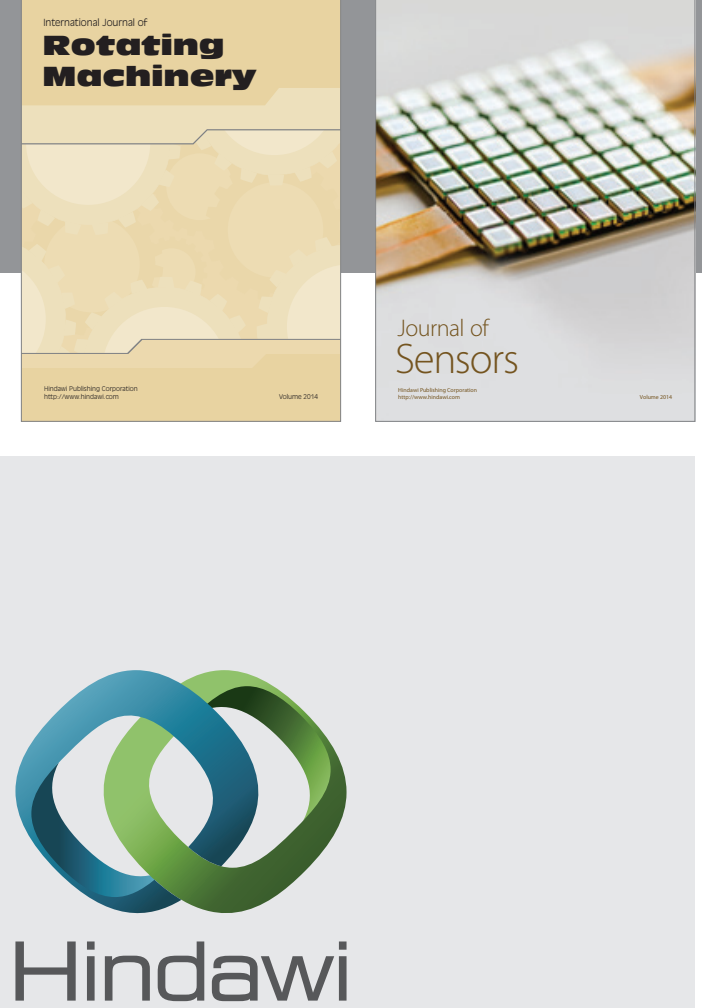

Submit your manuscripts at http://www.hindawi.com
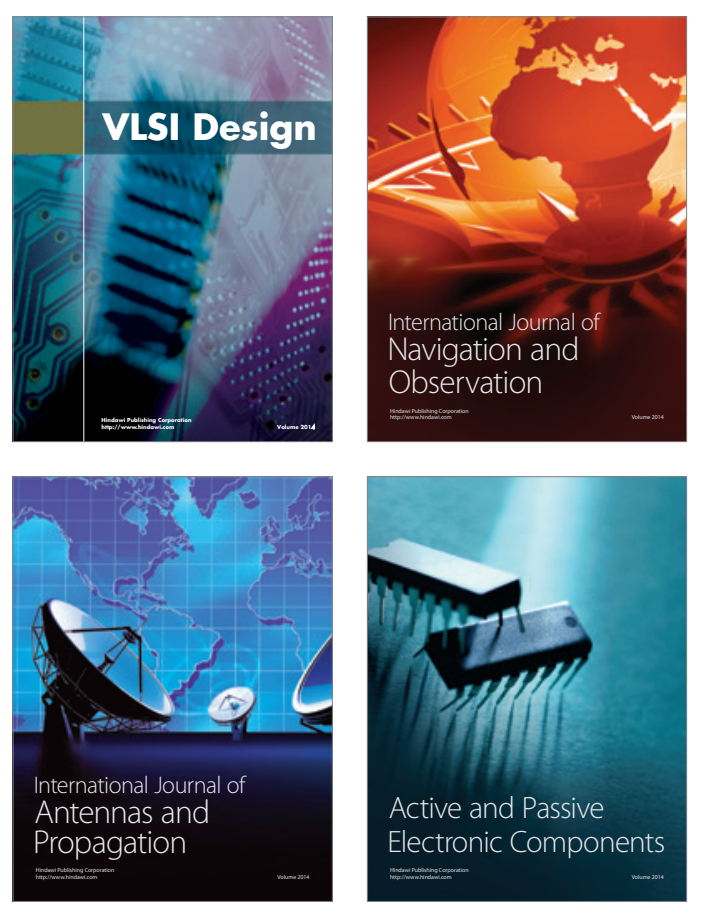
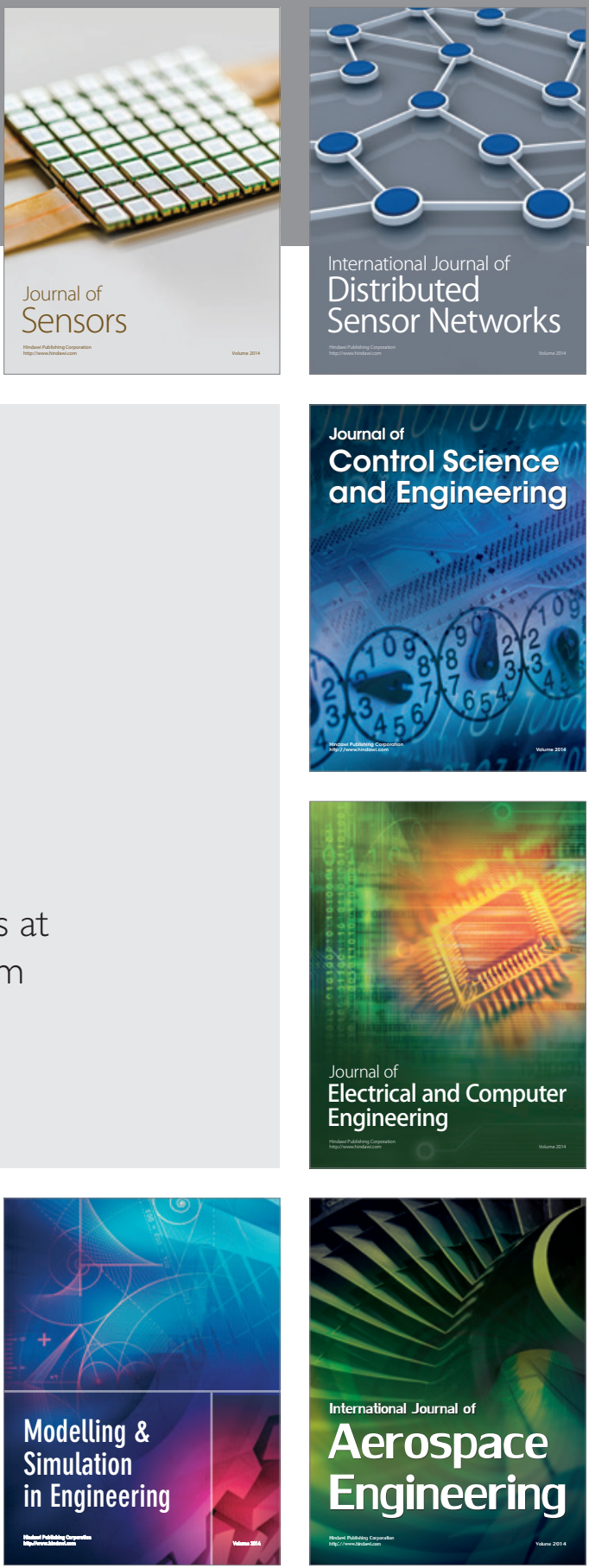

Journal of

Control Science

and Engineering
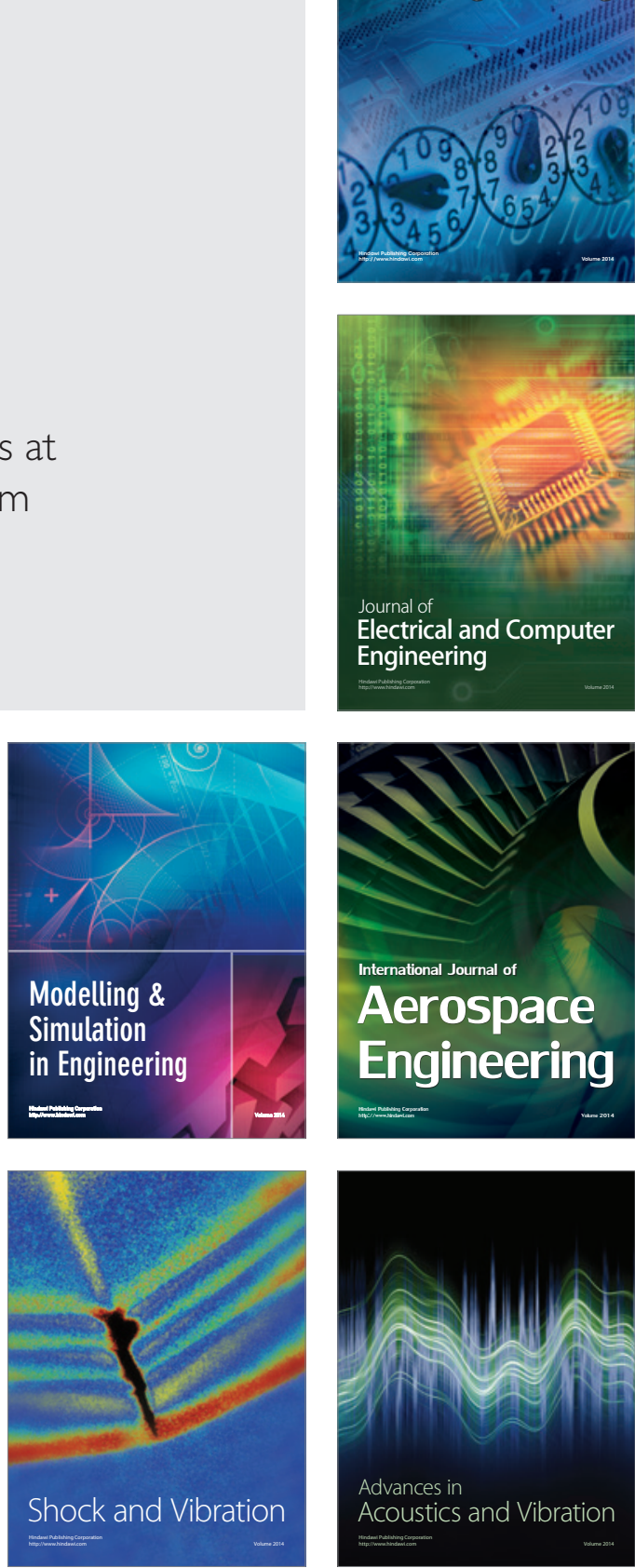